\title{
Construction of a didactic system for wave propagation speed determination in liquids
}

\author{
Francisco J. Arnoldำ, João L. S. P. de Oliveira ${ }^{1}$, Alexandre P. Silva ${ }^{1}$, Geraldo Dragoni Sobrinho ${ }^{2}$, \\ Cristhof J. R. Runge ${ }^{10}$, Marta S. G. Pires 2 20 \\ ${ }^{1}$ Universidade Estadual de Campinas, Faculdade de Tecnologia, Divisão de Telecomunicações, Limeira, SP, Brasil. \\ ${ }^{2}$ Universidade Estadual de Campinas, Faculdade de Tecnologia, Divisão Ambiental, Limeira, SP, Brasil.
}

Received on June 8, 2020. Revised on July 24, 2020. Accepted on August 6, 2020.

\begin{abstract}
This work shows the construction, calibration and validation of a system to determine sound speed in liquids. We used the pulse method for the measurements. Piezoelectric transducers are used to transmit and receive acoustic wave pulses. The pulse travel time is determined by a counter implemented in a logic programmable device (FPGA). Initially the system is calibrated using pure water. We performed measurements in $\mathrm{NaCl}$ and $\mathrm{CuSO}_{4}$ solutions at different concentrations. The system detected speed variations of approximately $1.0 \mathrm{~m} / \mathrm{s}$ in relation to the concentrations. Therefore, from didactical point of view, it is possible to perform experiments where small changes in the impurities concentration can be detected and measured. Further, the construction or even the enhancement of the system may be stimulant to students devoted to electronic instrumentation.
\end{abstract}

Keywords: Sound, Electronics, FPGA.

\section{Introduction}

Acoustic wave speed is an important parameter to investigate mechanical characteristics in materials. The speed is dependent on the elastic and the inertial properties and can be determined using non-destructive testing (NDT) [1]. Mechanical characteristics investigation has application in many areas such as Medicine [2] and food industry [3] to cite some.

Experiments for sound speed estimation in the air are popular in physics teaching labs. Usually, these experiments explore the resonances of stationary waves in tubes driven with tuning forks. However, despite of its importance, the measurements in liquids are not common due to the high cost of the experimental apparatus. The common approach is the theoretical one, and the speed of the acoustic wave in liquids is considered a constant value found in textbooks [4-6].

The methods for wave speed determination in liquids are classified in 3 groups [7]: I- phase velocity and group velocity; II - continuous wave method; III - pulse methods. The pulse methods are simple and popular, and although there are variations in these methods, they are based on square-pulse modulated sinusoidal sound waves, and subdivided in two categories. In the first, the sound waves are modulated with a square-pulse with duty cycle between 1 and $10 \mathrm{~s}$, and the system is considered narrowband. In the second, the pulses have less than $1 \mathrm{~s}$, and the transducers must respond in wideband.

*Endereço de correspondência: arnold@unicamp.br
The pulse method can be accomplished using piezoelectric transducers. Piezoelectric transducers can transmit and receive the waves [8]. Figure 1 and 2 show two possible configurations of an experimental system using piezoelectric transducers.

In transmit-receive configuration (Figure 1), one transducer is used as emitter (TE) and the other as receiver

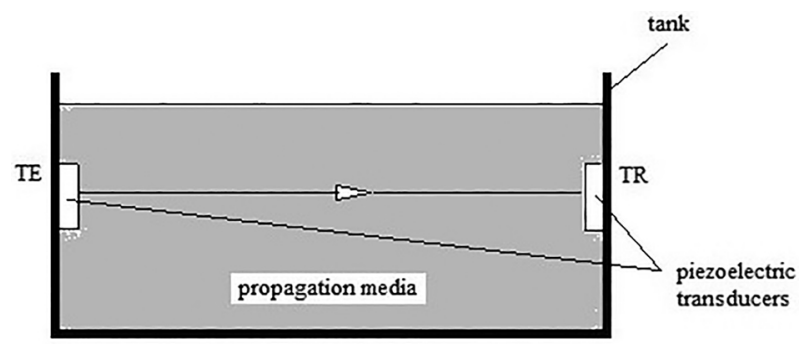

Figure 1: Experimental transmit-receive system to determine the wave propagation speed.

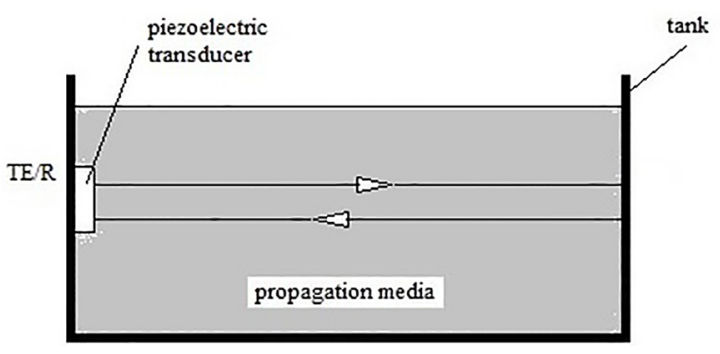

Figure 2: Experimental pulse-echo system to determine the wave propagation speed. 
(TR). TE and TR are positioned on the opposite walls of a tank containing the propagation medium. An electronic amplifier connected to TE generate electrical pulses. Via reverse piezoelectric effect, TE converts electrical pulses into acoustic waves that travel through the propagation medium and reach TR. In TR, the acoustic waves are converted into electrical pulses by the direct piezoelectric effect. Finally, TR is connected to a voltage amplifier that acts as signal conditioner for measurement or processing. The propagation speed of the waves is determined using Equation 1.

$$
v=\frac{\Delta s}{\Delta t}
$$

where $\Delta s$ is the distance between $\mathrm{T}$ and $\mathrm{R}$ (in $\mathrm{m}$ ), $\Delta t$ is the travel time of the acoustic waves (in s) and $v$ is the propagation speed wave of the media (in $\mathrm{m} / \mathrm{s}$ ).

In pulse-echo configuration (Figure 2) only one transducer acts as transmitter and receiver. Initially, an electronic system configures the transducer as transmitter (TE). The emitted waves travel through the propagation medium, reach a bulkhead and reflect back. During the travel time, the electronic system reconfigures the transducer as receiver (TR). Thus, when the waves reach the transducer, they are converted into the electrical signals and conditioned for measurement or processing. The propagation speed calculation is done using Equation 1, but considering twice the distance between transducers for $\Delta s$.

In both techniques described before, the wave travel time is a prime factor to determinate the propagation speed. However, in a teaching lab with limited resources, the travel time determination could be performed at most with a 2-channel oscilloscope. As a result in this case, using a common low-rate digital sampling oscilloscope and manually calibrated bar metering, the measurement accuracy can be impaired. Therefore, to improve accuracy, we replaced the traditional oscilloscope with a digital counter described in VHSIC Hardware Description Language (VHDL) and implemented in an FPGA. We have constructed a didactic, basic and low cost system for sound speed determination in liquids. Further, the simple technologic resources allow students understand, in a didactical way, the fundamental aspects of the sound speed measurements in liquids. Moreover, the system can be built by students with basic skill in electronic instrumentation.

We have constructed a system with two piezoelectric transducers (TE and TR), similar to system described in Figure 1, and with the respective electronic circuits for the signals processing. The precision is obtained with a state machine counter using a FPGA platform and a $50 \mathrm{MHz}$ clock. The wave travel time is determined counting the clock pulses during the travel time of the waves between TE and TR. This provides more precision than the conventional readings in oscilloscope screens. The system's simplicity make its construction and improvement affordable to students familiar with electronic instrumentation, in the same way that other similar systems developed in the past $[9,10]$ have been built and upgraded with new technologies.

This work is divided in five sections. Section II describes the basic concepts of the acoustic waves. Section III presents all parts of the developed system, comprising details and information need to reproduce and operate the system. Section IV presents the results obtained from the experimental tests and discussions. Section V concludes the article.

\section{Theory of speed wave propagation}

The acoustic waves are generated by a pressure perturbation in a volume element of the propagation medium. All propagation media have a bulk modulus [4-6], $B$ (in Pa), defined by Equation 2 .

$$
B=-\frac{\Delta p}{\Delta V / V}
$$

where $\Delta V / V$ is the relative variation of volume caused by pressure variation $\Delta p$ (in $\mathrm{Pa}$ ).

The minus sign in Equation 2 means that a pressure increase produces a volume decrease and vice versa. The wave propagation speed, $v($ in $\mathrm{m} / \mathrm{s})$, is determined by deducting the wave equation in an ideal fluid and is given by Equation 3

$$
v=\sqrt{\frac{B}{\rho}}
$$

where $\rho$ is the density of the propagation media (in $\left.\mathrm{kg} / \mathrm{m}^{3}\right)$.

Therefore, the wave speed in an ideal medium is dependent on the elastic and inertial properties of the propagation media. Although this theory covers all fluids, the system we have constructed only determines the speed in liquids. The reason for this limitation is that, in gases the impedance is low when compared with the piezoelectric ceramics. Therefore the impedance difference causes full reflections on the interface transducer-media, blocking any emission of acoustic energy. In solids the propagation of wave demands high voltages to excite the piezoelectric transducers. In addition, involves a more complex theory, not covered in introductory physics courses.

Finally, temperature variations, purity of the samples and dissolved air [11], are factor that may cause deviations in the measured speeds in liquids.

\section{Development of the electronic instrumentation and methodology}

In this section, we detail the development of the constructed system. Figure 3 shows a block diagram of the system as a whole.

The oscillator-amplifier (AE) generates an electrical pulse train that excites the transducer emitter (TE). The 


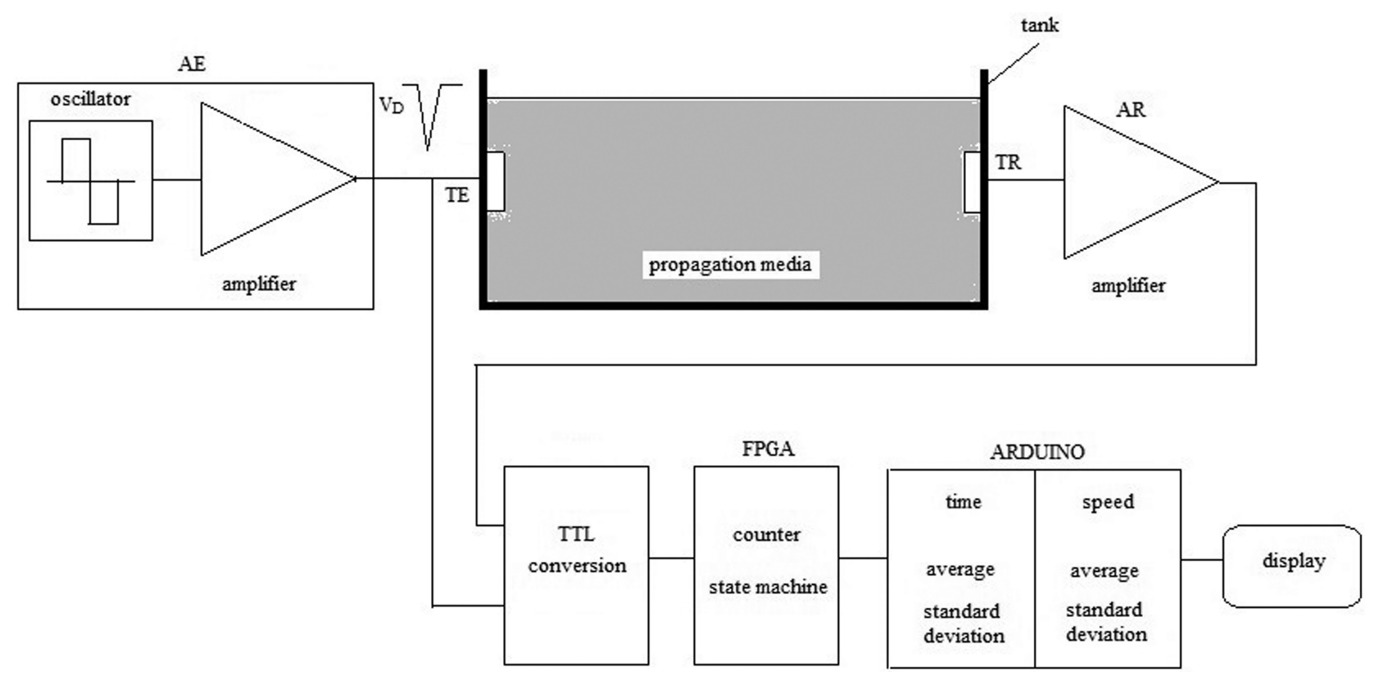

Figure 3: Block diagram of the constructed system.

transducer emits acoustic waves through propagation media. The waves are received by the transducer receiver (TR), and their acoustical energy are converted in a voltage. The voltage is amplified by an amplifier (AR) and conformed by a TTL converter. During the travel time of the waves through the media, an electronic counter implemented in a FPGA, counts the pulses of a $50 \mathrm{MHz}$ clock (used as a time base). For each propagated wave, the FPGA block provides the number of clock pulses counted between the emission and reception. The Arduino block calculates the wave propagation time using the clock pulse count provided by the FPGA block times the 50 $\mathrm{MHz}$ clock period (20 ns). Once the distance between TE and TR is known, speed is also calculated. Using consecutives samples in a predetermined fixed period of time, the mean and deviation of the propagation time and speed of the waves are calculated. Finally, a display shows the results.

\subsection{Construction of the transducers}

The transducers TE and TR were mounted with piezoelectric ceramics, fabricated by Eleceram Tech. Co. Ltda, Taiwan, disk-shaped, and are $25.4 \mathrm{~mm}$ (diameter) and $2.0 \mathrm{~mm}$ (thickness). The resonance of these ceramics at thickness vibration mode is $1 \mathrm{MHz}$. The flat surfaces of the ceramics are covered with silver electrodes where the wires for the connection with other circuits are welded. These ceramics were accommodated in PVC cylindrical tubes with external diameter of $32.0 \mathrm{~mm}$.

Figure 4 shows a schematic diagram of the transducer. On the rear part of the ceramic a backing layer is placed. The backing is comprised of glue (Araldite CY248 BR (65\%) and Aradur 837 BR (35\%)) and alumina powder (CT 3000, Almatis) in equal parts. Because of the high acoustic attenuation, the backing absorbs undesirable vibrations avoiding reflections and destructive interference of waves in the ceramic. Further, the backing decreases the transducer $Q$-factor allowing the emission and reception of waves at wideband. The frontal part of the

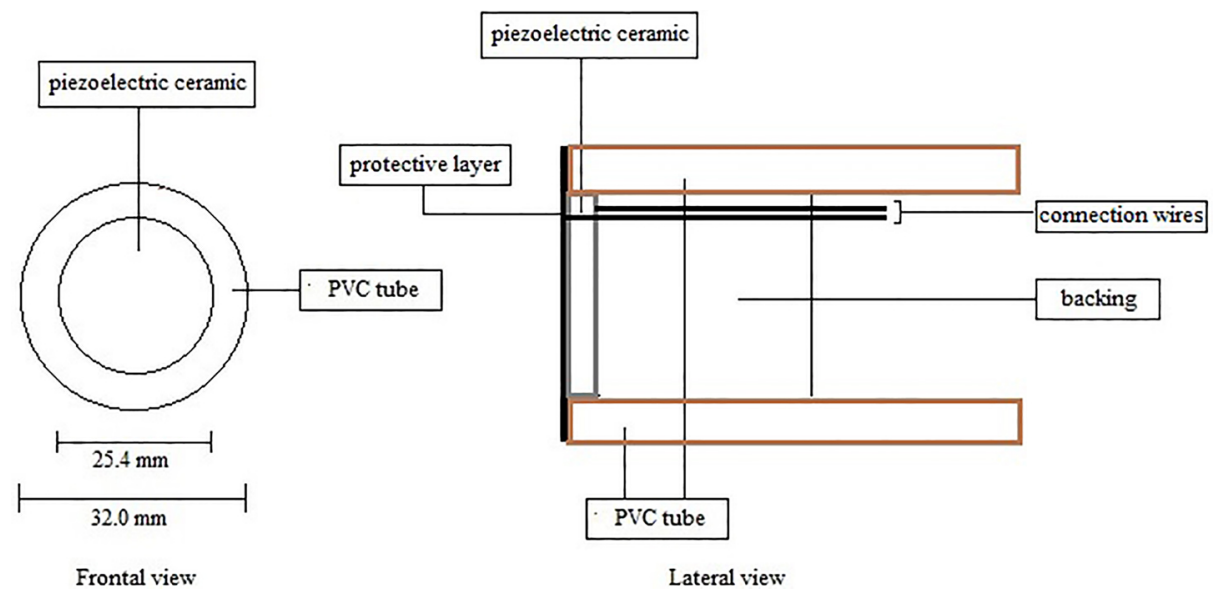

Figure 4: Frontal and lateral schematic view of the transducers. 


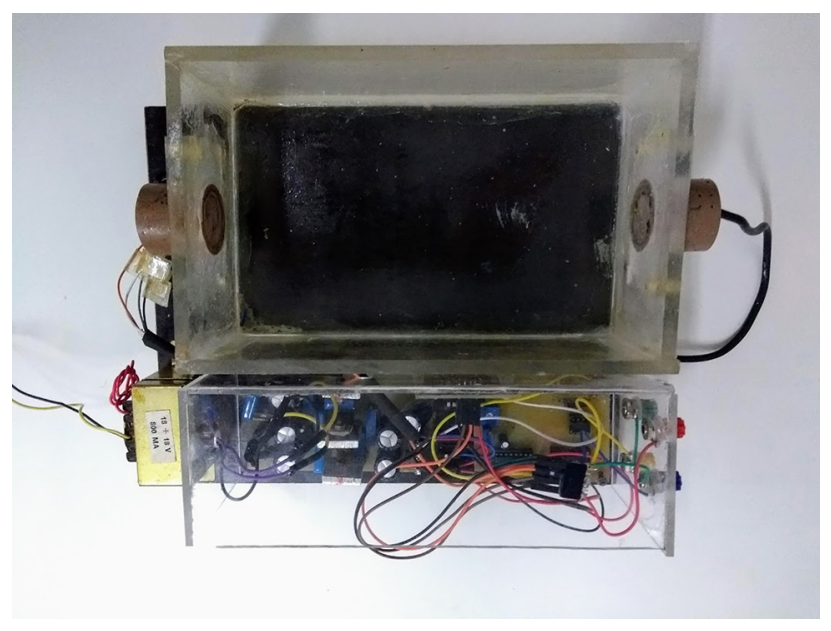

Figure 5: Photograph of test tank with transducers, and electronic circuits (voltage source, and emitter, receiver, and conversion circuits).

transducer is coated by a thin layer of plastic resin that protects the piezoelectric ceramic of the contact with the propagation media.

The transducers have been installed on the opposite walls of a tank made of acrylic with $192 \mathrm{~mm}$ (length), 117 $\mathrm{mm}$ (wide) and $68 \mathrm{~mm}$ (height) aligned to the length-axis. Figure 5 shows the photograph of the system comprising the tank and the transducers.

\subsection{Emitter circuit}

The electronic circuits to drive TE is based on Dorabiato work [12]. The emitter circuit consists of an oscillator, a power MOSFET amplifier and TE, as shown in Figure 6 . In the following the operation of emitter circuit is described.

First consider the MOSFET IRF740 in cutoff region (gate voltage, $V_{G}=0 \mathrm{~V}$ ). In this situation the source $(+\mathrm{HV})$ charges the $100 \mathrm{nF}$ capacitor with $12 \mathrm{~V}$. When a pulse is applied to the MOSFET gate by TPS2812, the device enters in saturation region and the capacitor discharges through the formed MOSFET channel. The capacitor discharge causes an abrupt variation in the voltage across TE, resulting in acoustic wave generation.

The periodic electric pulses are generated by the 74LS123 monostable multivibrators integrated circuit. The generated pulses have $5 \mathrm{~V}$ amplitude, 500 ns duration (adjusted by the $5 \mathrm{k} \Omega$ potentiometer) and $150 \mathrm{~Hz}$ frequency (adjusted by the $50 \mathrm{k} \Omega$ potentiometer). The TPS2812 receives the generated pulses and acts as high-speed current boosters to force the MOSFET saturation. While the pulse is high, the MOSFET remains saturated (500 $\mathrm{ns})$ and the $100 \mathrm{nF}$ capacitor discharges. When the pulse drops to a low-level, the MOSFET switch off (enter in cutoff region) and the $+\mathrm{HV}$ power supply recharges the $100 \mathrm{nF}$ capacitor until the next pulse (after 1/150 s). Finally, the acoustic waves generated due to the abrupt variation in the voltage across $\mathrm{TE}$, travel through the propagation medium and reach $\mathrm{TR}$ where the acoustic energy is converted into electrical signals.

\subsection{Receiver circuit}

The receiver circuit is also based on [12]. The converted signal of TR is amplified by an OPA820 operational amplifier. This integrated circuit is fundamental in the development of the project, because it is a wide band operational amplifier with a flat response up to high frequencies (hundreds of $\mathrm{MHz}$ ). The schematic diagram of the amplifier circuit is shown in Figure 7. In this circuit $\mathrm{R} 1=\mathrm{R} 3=10 \mathrm{k} \Omega$ and $\mathrm{R} 2=\mathrm{R} 4=100 \mathrm{k} \Omega$. Under these conditions the voltage gain is $\mathrm{VoR} / \mathrm{VTR}=\mathrm{R} 4 / \mathrm{R} 3=$ 10 , where $\operatorname{VoR}$ is the output voltage of the amplifier and VTR is the voltage produced at the receiving transducer. This amplifier is mounted in a small printed circuit board placed next to piezoelectric ceramic, inside the PVC tube, to avoid noise influence.

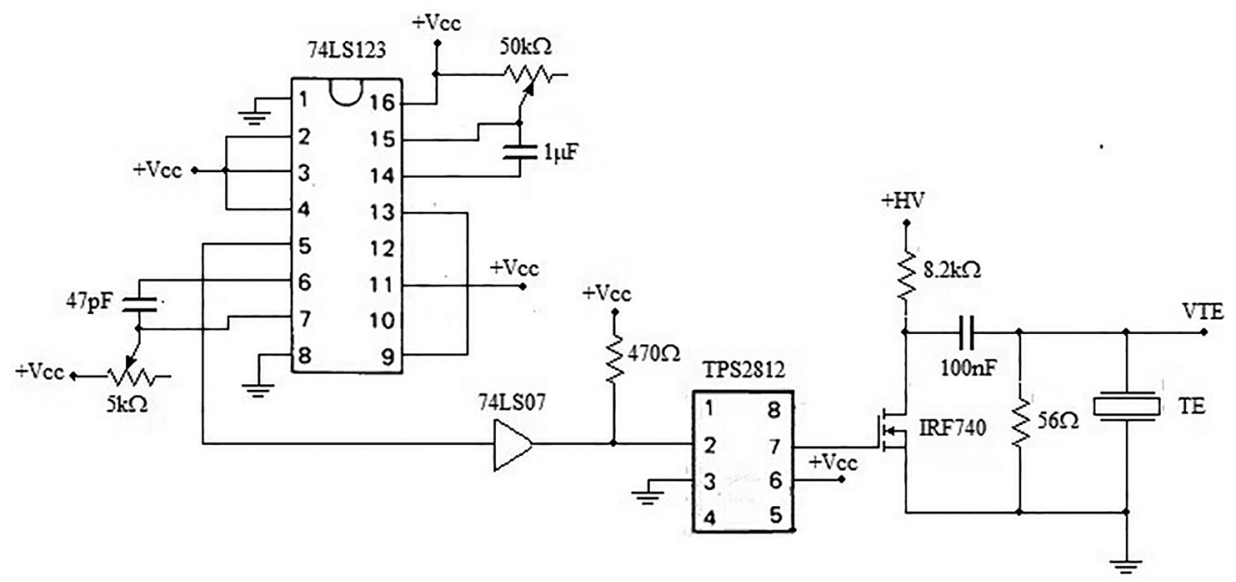

Figure 6: Schematics of emitter circuit. 


\subsection{Time counting and speed calculation}

Using a two channel oscilloscope, the emitted pulses by TE and received pulses by TR can be visualized connecting one channel to the MOSFET drain in circuit of Figure 6, and connecting other channel to the operational amplifier output in circuit of Figure 7 respectively. The wave propagation time through the propagation medium inside the tank can also be measure. Usually, standard digital oscilloscopes have measurement capabilities (cursors), allowing the use of manual bars or markers to determine the elapsed time between events. However, the adjustment resolution of these bars is often low, resulting in inaccurate time determination. Therefore, we decided to use a digital counter that counts the number of cycles of a $50 \mathrm{MHz}$ clock since the emission till reception of a pulse, incrementing every $20 \mathrm{~ns}$. The set-reset circuit used to enable and disable the counting is shown in Figure 8.

The voltages (VTE and VoR) from emitter and receiver circuits are converted in squared pulses by SchmittTrigger Inverter gates (7414) and set in TTL pattern. In the flip-flop RS (set by two NAND gates from a 7400), when the squared pulse relative to VTE goes to high level, the flip flop output is set (logic level 1). The flipflop remains this state until the squared pulse relative to VoR falls down, reseting it. Therefore, the travel time of the pulse is the interval while the flip-flop output is high.

Figure 9 shows the image file captured from the oscilloscope screen with the following signals: emitter circuit (drain of MOSFET, orange line), receiver circuit (VoR, blue line) and logic conversion covering the travel time (to FPGA, purple line). While the former signal is high, a counter implemented in a FPGA (a board with

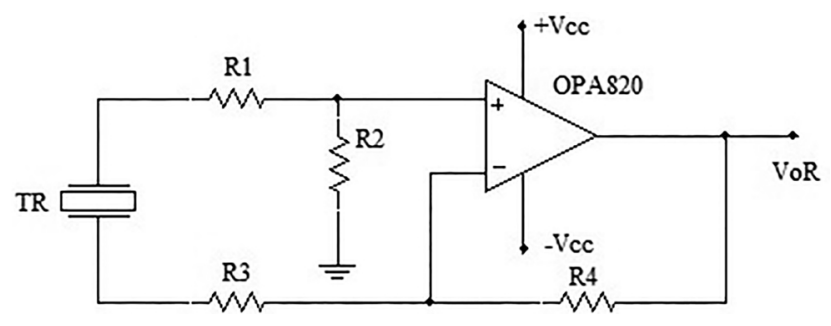

Figure 7: Schematics of the receiver circuit.

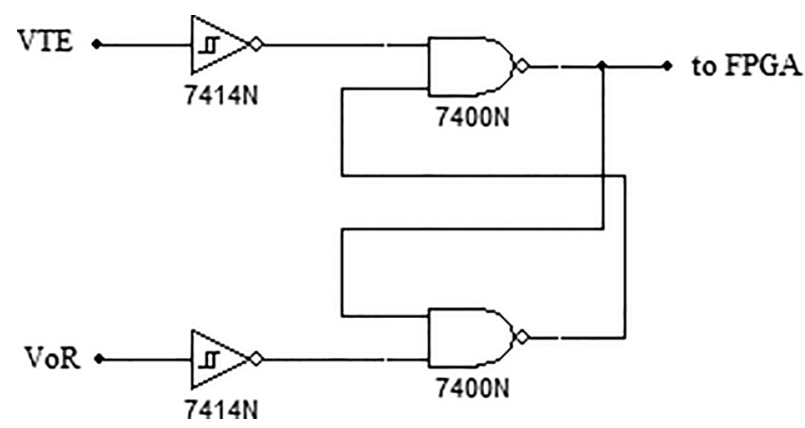

Figure 8: Circuit for the conversion from analogic to TTL pattern.

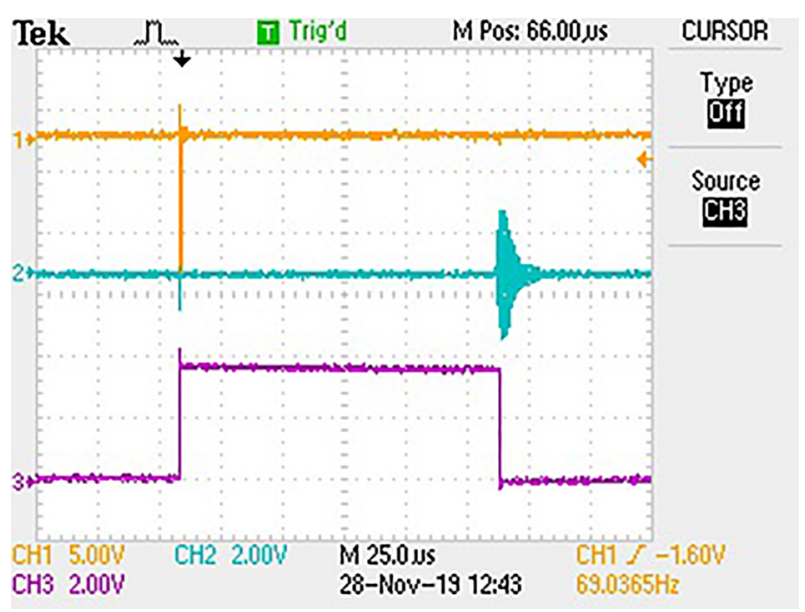

Figure 9: Oscilloscope screen of the captured voltages in drain of the MOSFET of the emitter circuit (orange line), output of the receiver circuit (blue line) and output of the conversion circuit (purple line).

on Cyclone IV FPGA (ep4ce6) and a $50 \mathrm{MHz}$ crystal oscillator clock [13]), performs the counting of clock cycles every 20 ns. The VHDL code implemented in the FPGA is found in https://github.com/RoosenRunge/ SoundSpeedLiquidSystem.

Since the TE transducer is periodically excited (150 $\mathrm{Hz}$ ), each transmitted pulse yields a TTL signal at logic level 1 which is used to determinate the speed of the wave in the propagation medium. Knowing that the distance between TE and TR is $19.2 \pm 0.5 \mathrm{~cm}$, the speed of waves in the fluid is calculated using Equation 1.

Using a fixed predetermined $N$ number of counts performed by the FPGA, the Arduino platform determines the mean and deviation values of the $N$ counts. With these values, the mean and deviation of travel time and the speed of wave are calculated. A display exhibits the mean and standard deviation obtained after calculation. Figure 10 shows the block diagram of the Arduino platform and the communication with FPGA (microcontroller ATMEGA 328A and module $\mathrm{I}^{2} \mathrm{C}$ ) and display. Two switches connected to the microcontroller are used to control the start of the reading and to select the nature of the reading (time/velocity). Implementations with Arduino are easily found in Internet [14]. The code we have used are also available in https://github.com/ RoosenRunge/SoundSpeedLiquidSystem .

Photographs of FPGA and Arduino boards are shown in Figures 11 and 12.

The mean $(\bar{t})$ and the standard deviation $(\sigma)$ are determined with Equation 4 and 5 .

$$
\begin{gathered}
\overline{\boldsymbol{t}}=\frac{\mathbf{1}}{\boldsymbol{N}} \sum_{\boldsymbol{i = 1}}^{\boldsymbol{N}} \boldsymbol{t}_{\boldsymbol{i}} \\
\sigma=\sqrt{\frac{\sum_{i=1}^{N}\left(t_{i}-\bar{t}\right)^{2}}{N-1}}
\end{gathered}
$$




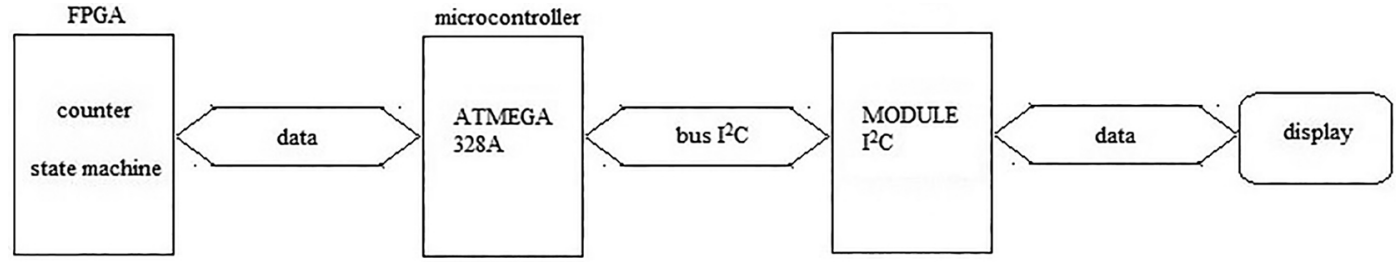

Figure 10: Block diagram of the interface to the display.

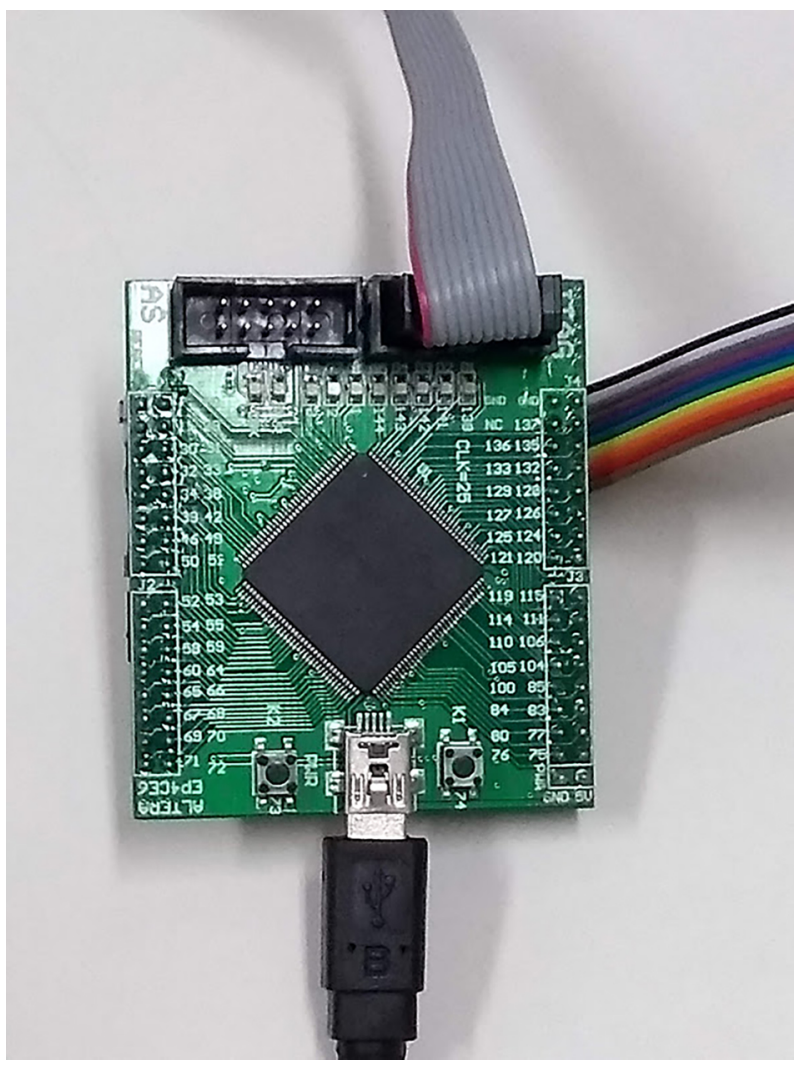

Figure 11: Photograph of the FPGA board (Cyclone IV FPGA (ep4ce6).

In our experiments pulses are generated every $6.67 \mathrm{~ms}$ $(150 \mathrm{~Hz})$. The value of $t_{i}$ (the time for a pulse to travel from TE to TR), is determined by the system, and the mean and standard deviation for travel time and the speed of waves, is calculated using $N=50$ samples.

\subsection{Calibration of the system}

The calibration of the system was performed using pure water obtained from a water purification system MilliQ Academic A10 (The LabWorld Group, USA). This system provides pure water with the following ASTM specifications:

Conductivity: $0.056 \mathrm{~S} / \mathrm{cm}$ in $25^{\circ} \mathrm{C}$;

Resistivity: $18 \mathrm{M} \Omega . \mathrm{cm}$ in $25^{\circ} \mathrm{C}$;

Total silica: $3 \mathrm{~g} / \mathrm{l}$

Total Organic Carbon (TOC): $100 \mathrm{~g} / \mathrm{l}$

Chlorides: $1 \mathrm{~g} / 1$

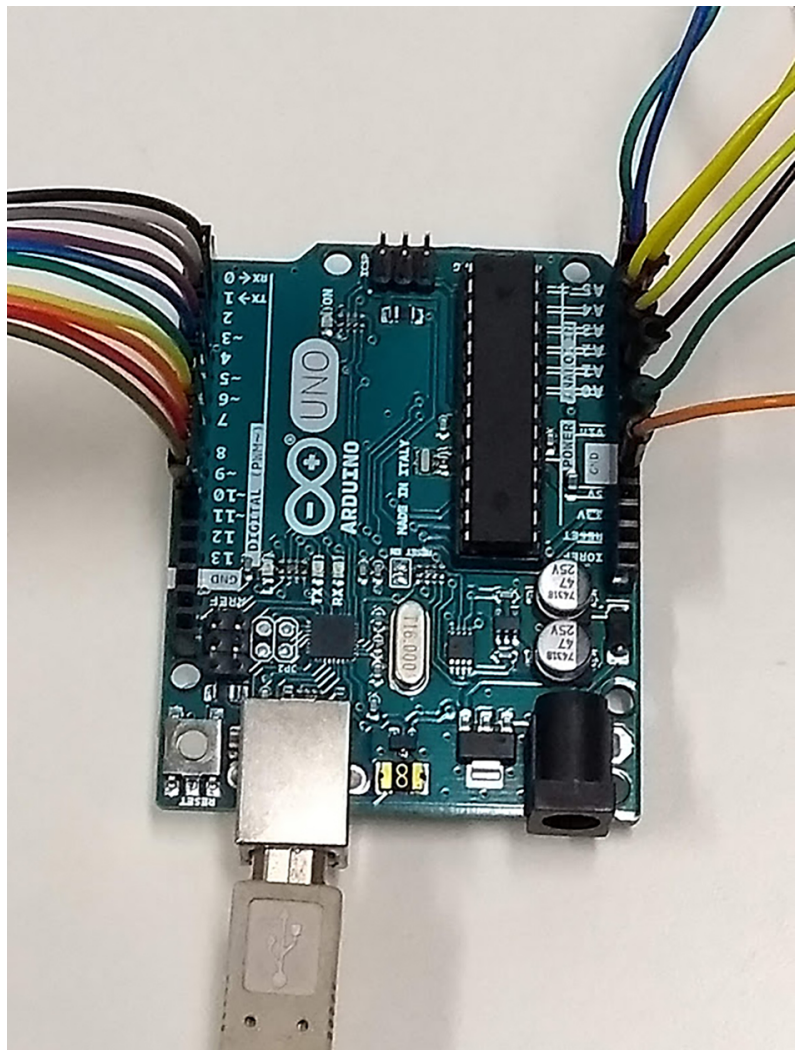

Figure 12: Photograph of Arduino board used in the system.

Sodium: $1 \mathrm{~g} / \mathrm{l}$

pH: not specified.

The tank was filled with $1.11 \mathrm{l}$ of pure water. A termocouple of a multimeter (Fluke 179) was also introduced in the tank to monitor the temperature. This termocouple was held on the bottom of the tank. At $26.6^{\circ} \mathrm{C}$, the value of sound speed measured using our system was $1469.81 \pm$ $3.94 \mathrm{~m} / \mathrm{s}$. This result was compared with the experimental polynomial approximation presented by Del Grosso [15] (Equation 6).

$$
v=k_{0}+k_{1} T+k_{2} T^{2}+k_{3} T^{3}+k_{4} T^{4}+k_{5} T^{5}
$$

where:

$v$ is the speed of sound of the pure water $(\mathrm{m} / \mathrm{s})$;

$T$ is the temperature of the liquid $\left({ }^{\circ} \mathrm{C}\right)$;

$$
\begin{aligned}
& k_{0}=0.140238754 \times 10^{4} \mathrm{~m} / \mathrm{s}, \\
& k_{1}=0.503711129 \times 10^{1} \mathrm{~m} / \mathrm{s} /{ }^{\circ} \mathrm{C}, \\
& k_{2}=-0.580852166 \times 10^{-1} \mathrm{~m} / \mathrm{s} /{ }^{\circ} \mathrm{C}^{2}, \\
& k_{3}=0.334198834 \times 10^{-3} \mathrm{~m} / \mathrm{s} /{ }^{\circ} \mathrm{C}^{3},
\end{aligned}
$$


$k_{4}=-0.147800417 \times 10^{-5} \mathrm{~m} / \mathrm{s} /{ }^{\circ} \mathrm{C}^{4}$,

$k_{5}=0.314643091 \times 10^{-8} \mathrm{~m} / \mathrm{s} /{ }^{\circ} \mathrm{C}^{5}$.

According to the Del Grosso approximation, the speed at $26.6{ }^{\circ} \mathrm{C}$ is $1500.87 \mathrm{~m} / \mathrm{s}$. A possible error in this measure may be caused by the delay in the switching of the electronic devices of the signal processing system. The delay effect has been compensated by adding $31.06 \mathrm{~m} / \mathrm{s}$ to the all results obtained through our system. Therefore, we predict an elapsed time of $6.18 \mathrm{~ms}$ to the switching signals of the electronics components.

\subsection{Experimental tests}

Initially, the experimental test determined the speed of waves in distilled water in a volume of $1.11 \mathrm{l}$. To verify speed variations of waves, different concentrations of sodium chloride $(\mathrm{NaCl}) \mathrm{P}$. A. was added in distilled water. We use solutions with different concentrations of $\mathrm{NaCl}$ until reach the salinity levels close to the sea water (around $35 \mathrm{~g} / \mathrm{l}$ ) [16]. The quantities of $\mathrm{NaCl}$ added to the initial distilled water volume to obtain the solutions were: $1,2,3,4,5,6,10,15,20,25,30$, and $35 \mathrm{~g}$. The water's temperature in this experiment was $22.6 \pm 0.1$ ${ }^{\circ} \mathrm{C}$.

To obtain the solutions with the predetermined quantities of $\mathrm{NaCl}$ in distilled water, the $\mathrm{NaCl}$ masses were determined using an analytical balance (Marte, AS5500C). After each solute adding, the solution was stirred during 30 s with a magnetic stirrer (Fisatom, 752, rotation of $750 \mathrm{rpm})$. Another solute, copper sulfate $\left(\mathrm{CuSO}_{4}\right.$ P.A.), was also used in other experiments applying the same procedure. The quantities of $\mathrm{CuSO}_{4}$ added to the initial distilled water volume of 1.111 were: $5,10,20,30,50,100$, 120 , and $150 \mathrm{~g}$. The water's temperature in experiments with $\mathrm{CuSO}_{4}$ was $23.1 \pm 0.1^{\circ} \mathrm{C}$. The experimental tests allow us to evaluate the sensitivity of the system considering the solution concentration used as propagation medium.

\section{Results and Discussion}

Figures 13 and 14 show the wave speed propagation measured in the experiments as a function of $\mathrm{NaCl}$ and $\mathrm{CuSO}_{4}$ concentration in distilled water, respectively.

Both results were fitted by a straight line (Equations 7 and 8 , respectively).

$$
\begin{aligned}
& v=9.2697 \times 10^{-4} C+1.4922 \times 10^{3} \\
& v=2.3025 \times 10^{-4} C+1.4942 \times 10^{3}
\end{aligned}
$$

where $C$ is the concentration of the solute in distilled water in $\mathrm{g} / \mathrm{l}$.

Therefore, we obtained sensitivities of $9.27 \times 10^{-4}$ $(\mathrm{m} / \mathrm{s}) /(\mathrm{g} / \mathrm{l})$ and $2.30 \times 10^{-4}(\mathrm{~m} / \mathrm{s}) /(\mathrm{g} / \mathrm{l})$ for the $\mathrm{NaCl}$ and the $\mathrm{CuSO}_{4}$, respectively. We observe that, with the same concentration dilution in distilled water, $\mathrm{NaCl}$ provides larger changes in the physical properties than the $\mathrm{CuSO}_{4}$.

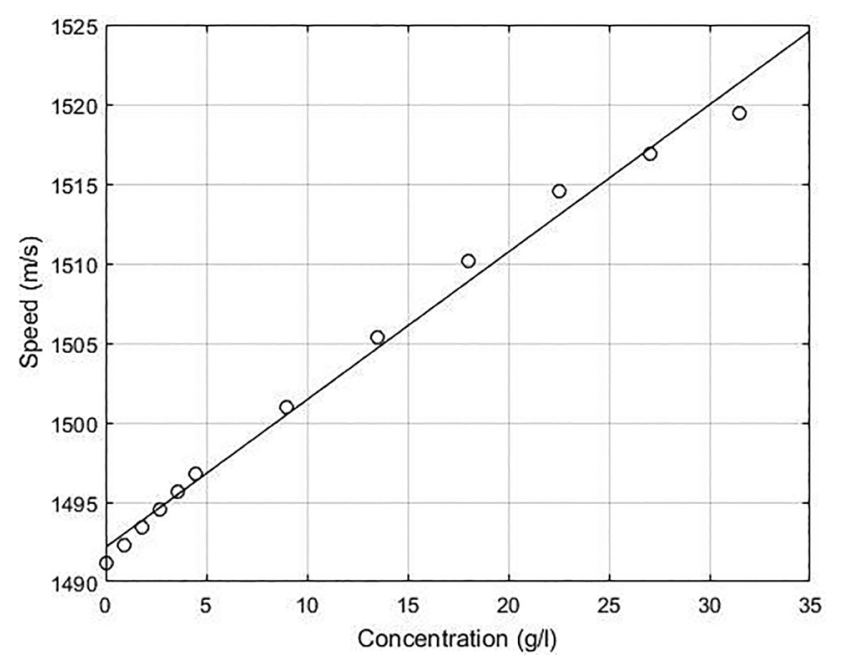

Figure 13: Speed wave as function of Concentration of $\mathrm{NaCl}$ in distilled water.

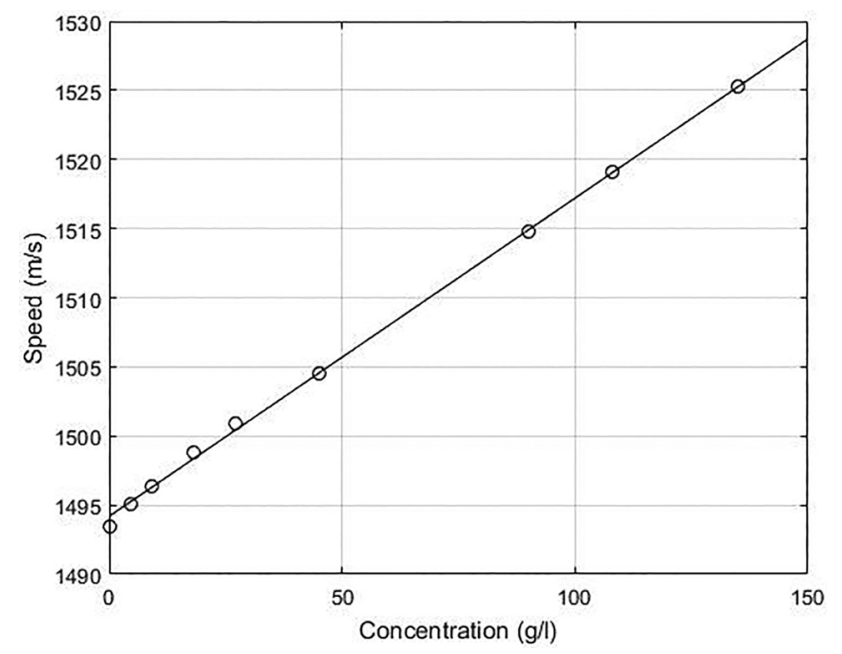

Figure 14: Speed wave as function of Concentration of $\mathrm{CuSO}_{4}$ in distilled water.

The standard deviation average of the speeds calculated using $N=50$ samples, were $4.68 \mathrm{~m} / \mathrm{s}$ and 3.94 $\mathrm{m} / \mathrm{s}$ for $\mathrm{NaCl}$ and $\mathrm{CuSO}_{4}$, respectively. We have detected speed changes around $1.0 \mathrm{~m} / \mathrm{s}$ from variations of $1.0 \mathrm{~g}$ in the $\mathrm{NaCl}$ mass addition. This result means that the measured time resolution is, at least, close to $0.1 \mathrm{~s}$.

The sound speed is dependent on intermolecular relations [17]. Therefore, each material presents a particular behavior. Thus, as a suggestion for teaching activities, the tests presented in this paper may be performed with other liquids to illustrate the determination of sound speed in liquids. Further, the temperature of the liquid causes variation on the sound speed, as observed in $[10,15]$. This issue will also be investigated in a future work as didactical aim. In this case, the experimental apparatus must be prepared to control the temperature and keeps it thoroughly homogeneous in the water tank. 
Temperature gradients may cause speed variations along the waves path.

Due to the attenuation of waves along the travel by the propagation medium, the use of the system to measure sound speed in fluids with high viscosity, such as glycerol, may not be performed with the current configuration of the equipment. Another issue is when the voltage generated by the receptor ( $\mathrm{TR}$ and $\mathrm{AR}$ ) has not enough amplitude for switching the Schmitt Trigger, and the pulse for FPGA to count, not triggers. This limitation can be overcome by either increasing the MOSFET shot voltage $(+\mathrm{HV})$, or by increasing the amplifier gain of the receptor circuit. These changes can still be used to extend the range of possible liquids used as propagation media.

Finally, the switching time of the electronic components affects the determination of the sound speed. This means, the system must use a reference liquid where the time of the acoustic pulse travel is at least of the same order of magnitude's sum of the electronic gates switching time used in the circuits. This reference liquid is necessary to the system calibration.

\section{Conclusion}

The experimental results show that the developed system can be used for the identification of small variations (as low as $1.0 \mathrm{~m} / \mathrm{s}$ ) of sound speed in distilled water and similar liquids. The low cost of this system (around US\$ 200) may stimulate students devoted to the development of electronic instrumentation to build and improve the current system, as well as create new systems based on this. In addition, this system can easily be operated by students of introductory semesters of exact and technological courses.

Finally, the developed system does not propose to be comparable or to have the same performance as highcost equipment used in high-precision laboratories, but to allow educational experiments to measure variations in the speed of sound in liquids, in didactic activities. The students may construct in teaching labs and be stimulated to create experiments where the variations of speed can be detected from temperature changes and addition of impurities. The experiments performed with the developed system facilitate the understanding of sound speed concept showing it as a factor dependent on the inertial and elastic properties changes.

\section{Acknoledgements}

The authors are grateful to FAPESP (The São Paulo Research Foundation - Brazil (2012/07639-4)) and UNICAMP (State University of Campinas - Brazil) by the financial support.

\section{References}

[1] J. Krautkramer and H. Krautkramer, Ultrasonic testing of materials (Springer-Verlag, Berlim, 2013).

[2] P.N.T. Wells, Biomedical Ultrasonics (Academic Press, London, 1977).

[3] T.S. Awad, H.A. Moharram, O.E. Shaltout, D. Asker and M.M. Youssef, Food Research International 48, 410 (2012).

[4] D. Halliday, R. Resnick and J. Walker, Fundamentals of Physics (Willey, Cleveland, 2011), v. 2, $9^{\text {th }}$ ed.

[5] P.A. Tipler and G. Mosca, Physics for Scientists and Engineers (Freeman, New York, 2007), v. 1, $6^{\text {th }}$ ed.

[6] P.R. Kesten and D.L. Tauck, University Physics for Physical and Life Sciences (Freeman, New York, 2012), v. 2.

[7] U. Kaatze, F. Eggers and K. Lautscham, Meas. Sci. Technol. 19, 062001 (2008).

[8] A. Arnau, Piezoelectric Transducers and Applications (Springer, Berlin, 2008), $2^{\text {nd }}$ ed.

[9] G.W. Finken and E.A. Hiedmann, J. Acoust. Soc. Am. 28, 921 (1956).

[10] N.P. Cedrone and D.R. Curran, J. Acoust. Soc. Am. 26, 963 (1954).

[11] A.J. Barlow and E. Yazgam, Brit. J. Appl. Phys. 17, 807 (1966).

[12] L.F. Dorabiato, Sistema de ultra-som para caracterização de leite bovino. Masters Dissertation, Universidade Tecnológica Federal do Paraná, Curitiba (2007).

[13] C.J.R. Runge, M.L. Cerqueira and F.J. Arnold, Rev. Bras. Ens. Fis. 42, e20190208 (2020).

[14] https://www.arduino.cc/, accessed in 27/02/2020.

[15] V.A. Del Grosso and C.W. Mader, J. Acoust. Soc. Am. 52, 1442 (1972).

[16] K.W. Mackenzie and J. Acoust. Soc. Am. 70, 807 (1981).

[17] B. Jacobson, J. Chem. Phys. 20, 927 (1952). 